\title{
A SEARCH FOR COUPLED-BUNCH INSTABILITY IN THE FERMILAB MAIN INJECTOR
}

\author{
C. M. Bhat, \\ FNAL*, Batavia, IL 60510, USA
}

\begin{abstract}
In the Fermilab Main Injector (MI) we are planning to double the bunch intensity from its design value by slip stacking. The accelerator consists of $18 \mathrm{rf}$ cavities which operate at a harmonic number of 588. These cavities are known to have many higher ordered resonances. Longitudinal coupled-bunch instability induced by excitation of the rf cavities may be an important issue to be addressed in the intensity upgrade. Here we have carried out some simulation studies of the longitudinal coupled bunch instability to investigate bunch intensity limits. The results are presented in this paper.
\end{abstract}

\section{INTRODUCTION}

The Fermilab Main Injector[1] is a high energy, high intensity proton synchrotron. The maximum design beam intensity of MI is 5E12/Booster batch of 84 bunches (of $53 \mathrm{MHz}$ rf system) and/or 3E13 protons per machine cycle (which requires six Booster batches). In recent years, due to many proposals of MI fixed target high energy physics experiments, the demand for the $120 \mathrm{GeV}$ protons has increased considerably. Therefore, we are planning to upgrade the MI beam intensity by at least a factor of two using the method of slip stacking[2]. MI rf cavities, however, are known to have many higher order resonances and coupledbunch instability induced by excitation of these resonances may become an important issue to be addressed in the intensity upgrade. Here we present results of beam dynamics simulations studies of coupled bunch instability in the MI.

\section{LONGITUDINAL BEAM DYNAMICS CALCULATIONS}

As a proton bunch passes through an rf cavity during acceleration or storage, it excites resonant modes with high Q-values and high Rs (shunt impedance), leaving energy in the cavity. The excitation amplitude depends upon the bunch intensity. If this excitation persists until the arrival of subsequent bunches, one may expect coherent build up of synchrotron motion of bunches i.e., longitudinal coupledbunch instability[3], may be induced. Coupled-bunch motion might continue to grow exponentially until a mechanism such as Landau damping limits further growth. However, this motion of bunches inside a bucket would lead to emittance growth. The source of bunch emittance growth of this nature would certainly depend upon bunch number as

\footnotetext{
* Work supported by the U.S. Department of Energy under contract No. DE-AC02-76CH03000.
}

well as number of bunches in the synchrotron. In general, one might expect excitation of transverse as well as longitudinal instabilities due to different cavity modes, however, here we study only the longitudinal coupled bunch instability in the MI.

The longitudinal coupling impedance $Z_{\|}(\omega)$, at a frequency $\omega$, is given by

$$
Z_{\|}(\omega)=-\frac{V_{\|}(\omega)}{I_{B}(\omega)}
$$

where $V_{\|}(\omega)$ is the longitudinal voltage per turn induced in the beam and $I_{B}(\omega)$ is the Fourier amplitude of the beam current.

The impedance due to the rf cavities may consist of sharp peaks at resonant frequencies corresponding to the cavity mode and will be driven at higher order parasitic modes.

The longitudinal component of the impedance caused by any mode is

$$
Z_{\|}(\omega)=\frac{R_{S}}{\left[1+j Q\left(\frac{\omega_{R}}{\omega}-\frac{\omega}{\omega_{R}}\right)\right]}
$$

In this equation $\omega_{R}=2 \pi f_{R}$ is the resonance angular frequency and $R_{S}$ is expressed in ohms.

For $M$ equally spaced coupled bunches there are $M$ possible dipole modes labeled by $m=1,2, \ldots, M$. With the azimuthal position of the centroid of each bunch, $\theta_{l}, l=$ $1,2, \ldots, M$. The signature of the simplest coupled bunch mode is given by

$$
\theta_{l}(t)=\theta_{0} \sin \left(2 \pi m l / M-\omega_{s} t\right)
$$

and has the form of a discrete propagating plane wave. In addition, an individual bunch in the $m$-th coupled bunch mode requires an index " $n$ " to describe its motion in synchrotron phase space , e.g., the dipole mode, $n=1$, where the bunches move rigidly as they execute longitudinal synchrotron oscillations.

A simple resonant condition for the mth dipole mode driven by the longitudinal impedance $Z(\omega)$ sharply peaked at $\omega_{c}=(n M+m) \omega_{o} \pm \omega_{s}$ where $\omega_{o}$ is revolution frequency and $n$ is an integer. From this we can see that the resonance condition exists on a limited time interval.

In the past, many higher order resonances have been observed in the MI rf cavities [4]. The measured modes of the cavity without cavity bias and with cavity bias are shown in Fig. 1. Passive and active dampers have been developed to reduce the effect of some of the important modes viz., one at $127 \mathrm{MHz}$ and another at $227 \mathrm{MHz}$. 
Recently, during the 1996-98 Fermilab fixed target experiment, I have observed a resonance at about $82 \mathrm{MHz}$ in some of the cavities which starts contributing to the total impedance. This observation was made in the old Fermilab Main Ring synchrotron with 1008 bunches in the machine with an average bunch intensity of $2.48 \times 10^{10}$ protons. Now in the MI we are using the same set of $18 \mathrm{rf}$ cavities but with almost the double the Main Ring bunch intensity (and plan to quadruple the bunch intensity for the future use). Under these new operating conditions, this resonance might introduce instability in the beam. In the present investigation, we select four prominent modes (Table 1, resonances frequencies with “*”) below $350 \mathrm{MHz}$ and tried to study the effect of all of them in accelerating 84 bunches in the Main Injector. The Q-values and shunt impedances (measured with with active dampers for $127 \mathrm{MHz}$ and 227 MHz resonances) are listed in Table 1. The study of coupled bunch instability is carried out using multi-particle longitudinal beam dynamics simulation computer code ESME [5].

Table 1: Shunt Impedance in the FMI cavities

\begin{tabular}{ccc}
\hline frequency(MHz) & Impedance $(\Omega)$ & $\mathrm{Q}$ \\
$f_{R}$ & $R_{S}$ & \\
\hline $82.253^{*}$ & 20980.0 & 2734 \\
$127.25^{*}$ & 7140.0 & 241 \\
197.776 & 944.4 & 100 \\
$227.549^{*}$ & 20790.0 & 456 \\
261.996 & 1041.0 & 100 \\
$336.876^{*}$ & 987.0 & 687 \\
(Broad Band - beam pipe & & \\
Impedance) & & \\
$2.177 \mathrm{E} 03$ & $0.72282 \mathrm{E} 5$ & 1 \\
\hline
\end{tabular}

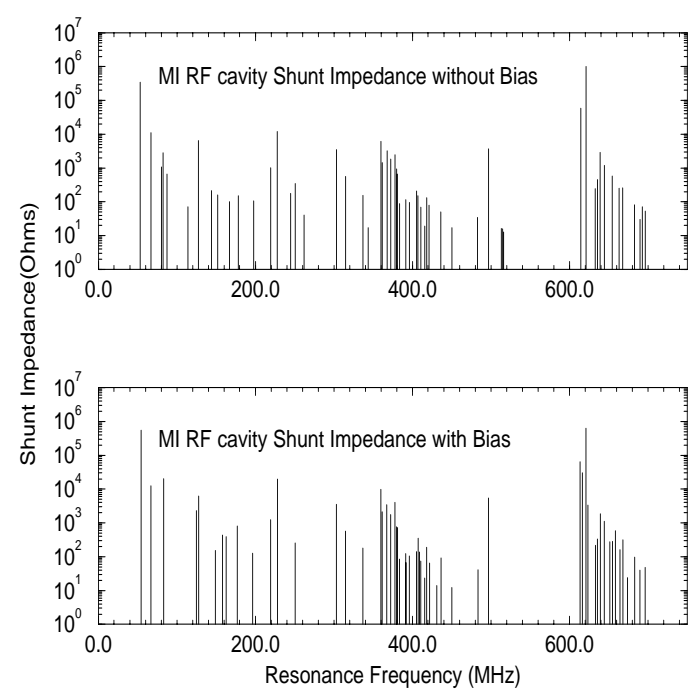

Figure 1: Shunt Impedance vs. Frequency[4].
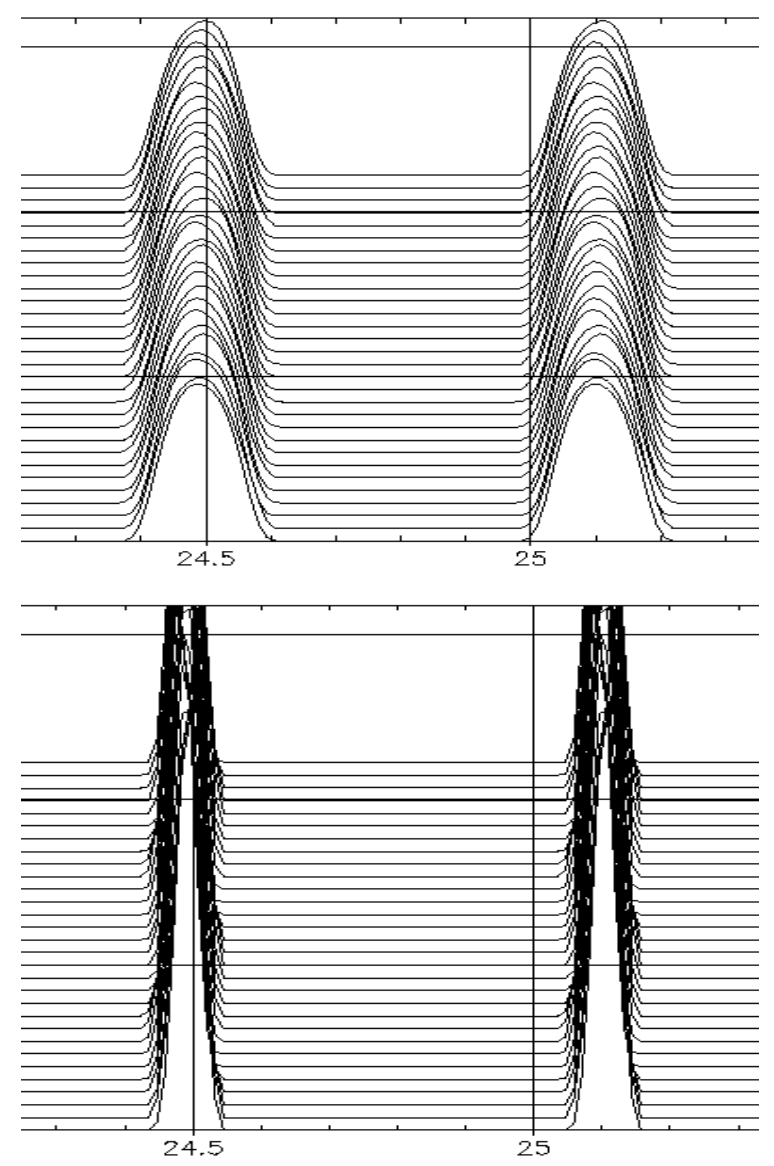

Figure 2: Simulated mountain range plots for the last two bunches in a train of 84 bunches in the Main Injector at injection energy (top) and Soon after transition (bottom). Each trace is with a spacing of $0.33 \mathrm{~ms}$. The top

\section{ESME SIMULATIONS AND RESULTS}

MI is nearly a circular accelerator with an average radius $\mathrm{R}=528.302$ meters. The longitudinal particle tracking code ESME is used to study time evolution in phase space of a beam undergoing instabilities in the MI. The tracking procedure used is a turn by turn iteration of two Hamilton-type difference equations describing synchrotron oscillation in $\theta-\epsilon$ phase space, where $\theta$ is azimuthal phase angle and $\epsilon$ is deviation from the synchronous phase angle. In order to include the effect of the beam environment one can consider the additional potential due to the wake field generated by the beam as it passes through the vacuum pipe. Knowing the particle distribution in the azimuthal direction $\rho(\theta)$, one can construct a wake field induced voltage as follows:

$$
V(\theta)=e \omega_{0} \sum_{k} \rho_{k} Z\left(k \omega_{0}\right) e^{j k \theta}
$$

where $\rho_{k}$ represents the discrete Fourier spectrum of the beam and $Z(\omega)$ is the longitudinal coupling impedance. 
The equations used by ESME are:

$$
\begin{aligned}
\theta_{i, k} & =\left[\frac{\tau_{s, k-1}}{\tau_{s, k}} \theta_{i, k-1}+2 \pi\left(\frac{\tau_{i, k}}{\tau_{s, k}}-1\right)\right]_{\bmod (\pi)} \\
E_{i, k} & =E_{i, k-1}+e V\left(\phi_{s, k}+h \theta_{i, k}\right)-e V\left(\phi_{s, k}\right)
\end{aligned}
$$

where, $\tau_{s, k}$ is the circulation period for kth turn of synchrotron particle and $\theta_{i, k}$ is referred to as particle azimuth, $\mathrm{E}$ refers to energy and $\phi_{s, k}$ is synchronous phase. The results may be viewed in time domain or in frequency domain, passing from one to the other through Fast Fourier Transforms.

The space charge effect is included in the simulation, which has the form

$$
\left(\frac{Z_{\|}}{n}\right)^{\text {space-charge }}=i \frac{377}{2 \beta \gamma^{2}}\left[1+2 \ln \frac{b}{a}\right][\text { Ohm }]
$$

where $b=0.058 \mathrm{~m}$ is the average beam pipe radius and $a=0.005 \mathrm{~m}$ is the average beam size, $\beta$ and $\gamma$ are the relativistic quantities $v / c$ and $E / m_{o} c^{2}$, respectively. Other relevant parameters are listed in are taken from the ref.1.

The impedance data shown in Table 1 are from the measurements on one of the rf cavities. All eighteen rf cavities have similar structure. Hence, in our simulations we use the same $R s$ and Q-values listed in Table 1 for each of the $18 \mathrm{rf}$ cavities. The broad band impedance of the MI is assumed to be $3 \Omega$, with a safety factor of two.

ESME calculations have been carried out on two configurations of bunches, viz, one Booster batch of 84 bunches and 6 Booster batches to a total of 504 bunches in the accelerator. In both cases we take 2000 macro-particles per bunch, where each particle is assumed to have an electronic charge of $3 \times 10^{7} e$, for simulation. The tracking is carried out from injection momentum of $8.889 \mathrm{GeV} / \mathrm{c}$ to 120 $\mathrm{GeV} / \mathrm{c}$. The first case is important for the $\bar{p}$ production cycle, and the second scenario is relevant to the fixed target operation mode of the MI.

The simulation results are presented in Fig. 2 for the case of 84 bunches in the MI. The Figure on the top shows the predicted bunch profiles for every $330 \mu$ s at $8 \mathrm{GeV}$ for bunch number 82 and 83 . We observe small oscillations on these bunches with an amplitude of oscillation $i 12^{\circ}$ (in rf phase). Ultimately this might lead to an emittance growth of about $7 \%$ Simulations also showed similar oscillations arising from transition crossing which will damp away later on the cycle. However, in no calculations we have seen any higher order mode coupled bunch excitations during acceleration or elsewhere. In our calculations we scanned up to $12 \mathrm{E} 10$ protons/bunch which is twice the MI design intensity.

In conclusion, our simulations show that we can up-grade the beam intensity in the MI by a factor of two from its design bunch intensity. In the case of 84 bunches in the MI at most $10 \%$ emittance growth is expected due to coupledbunch instability induced by the MI rf cavity higher order mode excitations. This has practically no effect on pbar stacking. The measurements with beam in the MI with bunch intensity very close to the design intensity of $6 \mathrm{E} 10$ protons/bunch also show similar behavior. However, our preliminary simulations show signatures of coupled bunch instability for the case of 504 bunches in the MI[6]. Detailed study is needed to confirm these results.

The author would like to thank Jim MacLachlan for his help in ESME program and many useful discussions. I also would like to thank Dave Wildman and Joe Dey for providing me with cavity resonance data. Assistance of Mrs. F. Rivera-Medero in running some early simulations is acknowledged.

\section{REFERENCES}

[1] Fermilab Main Injector Technical Design Report (1994).

[2] S. Shukla et. al., Proceedings PAC97, page 144 (1997).

[3] S. Stahl and S.A. Bogacz, Phys. Rev. D 37, 5 (03/1988), pp 1300-1036, and references there in.

[4] J. Dey and D. Wildman, Proceedings of the IEEE, 06/96, pp 1675-1677

[5] J. A. MacLachlan, FN-446, Feb/1987, and J. MacLachlan and J.-F. Ostiguy, Enhancements to ESME

[6] "Coupled-Bunch Instability in the Fermilab Main Injector: Longitudinal Phase Space Simulation," F. Rivera-Medero and C.M. Bhat,Fermilab SIST Report (unpublished) 1998. 